\title{
DIMENSION OF DENSE SUBALGEBRAS OF $C(X)$
}

\author{
JUAN B. SANCHO DE SALAS AND MARIA TERESA SANCHO DE SALAS
}

(Communicated by Dennis K. Burke)

\begin{abstract}
The real spectrum of any R-algebra $A$ is the set of all maximal ideals of $A$ with residue field $\mathbf{R}$, endowed with the initial topology for the functions induced by the elements of $A$. We prove that a compact metric space $X$ has dimension $\leq n$ if and only if $X$ is the real spectrum of an algebra of Krull dimension $\leq n$; so that the dimension of $X$ is the minimum of the Krull dimensions of all dense subalgebra of $C(X)$. Moreover, we prove that a compact Hausdorff space $X$ has covering dimension $\leq n$ if and only if every countably generated subalgebra of $C(X)$ is contained in the closure of a subalgebra of Krull dimension $\leq n$.
\end{abstract}

\section{INTRODUCTION}

The aim of this work is to determine the dimension of any compact Hausdorff space $X$ in terms of the Krull dimension of dense subalgebras of $C(X)$.

In Algebraic Geometry, the dimension of an affine algebraic variety $V$ is defined as the Krull dimension of the ring $A$ of all algebraic functions on $V$ (i.e. the affine coordinate ring):

$$
\operatorname{dim} V=\operatorname{dim} A=\left[\begin{array}{l}
\text { Supremum of the lengths of all } \\
\text { chains of prime ideals in } A
\end{array}\right] .
$$

Moreover, Noether's Lemma states that $\operatorname{dim} V \leq n$ if and only if there exists a finite morphism from $V$ to the affine $n$-space (i.e. a finite morphism $\left.k\left[x_{1}, \ldots, x_{n}\right] \rightarrow A\right)$.

In Topology, the equivalent result to Noether's Lemma is simply Katětov's characterization of the dimension: A compact metric space $X$ has dimension $\leq$ $n$ if and only if there exists a continuous map $X \rightarrow \mathbf{R}^{n}$ with totally disconnected fibers. In terms of $C(X)$, we have $\operatorname{dim} X \leq n$ if and only if there exists a morphism $\mathbf{R}\left[x_{1}, \ldots, x_{n}\right] \rightarrow C(X)$ such that its image is an analytic base for $C(X)$. By a suitable modification of the statement, this characterization may be extended to arbitrary compact Hausdorff spaces.

Received by the editors September 30, 1987 and, in revised form, February 8, 1988.

1980 Mathematics Subject Classification (1985 Revision). Primary 54C40, 54F45.

Key words and phrases. Dense subalgebras of $C(X)$, Krull dimension, real spectrum, inverse limits of polyhedra, covering dimension. 
Our main result, which may be considered as analogous to $(*)$ in Topology, is the following theorem:

Theorem. A compact metric space has dimension $\leq n$ if and only if it is the real spectrum of an algebra of dimension $\leq n$.

In other words, the dimension of any compact metric space $X$ is the minimum of the Krull dimensions of all dense subalgebras of $C(X)$.

We hope that this characterization may be extended to arbitrary compact Hausdorff spaces replacing the covering dimension by the arithmetical dimension. However, we obtain the following characterization for the covering dimension: A compact Hausdorff space $X$ has covering dimension $\leq n$ if and only if every countably generated subalgebra of $C(X)$ is contained in the closure of a subalgebra of Krull dimension $\leq n$.

\section{DiMENSION OF TOPOLOGICAL SPACES}

We shall use a definition of dimension, inspired by the Krull dimension of rings, which coincides with the inductive and covering dimensions for separable metric spaces. This dimension was set up by J. B. Sancho Guimerá during the seventies and the corresponding dimension theory was developed by R. Galián in [4].

Let $X$ be a topological space. The family $A_{X}$ of all closed sets in $X$, with the addition and the product defined as the intersection and the union respectively, is almost a commutative ring (only the existence of inverse elements relative to the addition fails) where $0=X$ and $1=\varnothing$.

Definition 2.1. We shall say that a set $A$ with two binary composition laws (addition and product) is a distributive lattice if it is a commutative semigroup under both operations, the product is distributive over the addition and for any $a \in A$ we have

$$
a \cdot 0=0, a^{2}=a, \quad 1+a=1 .
$$

It is clear that the family $A_{X}$ of all closed sets in a topological space $X$, with + and - interpreted as intersection and union respectively, is a distributive lattice. However, note that distributive lattices are not assumed to have arbitrary sums. We use the algebraic notation + and - instead of the usual symbols $\vee$ and $\wedge$ because the definition of dimension is inspired by the Krull dimension of rings.

We shall say that a subset $B$ of a lattice $A$ is a sublattice if it is closed under both operations and 0,1 belong to $B$. A nonempty subset $I$ of $A$ is said to be an ideal (most authors call it filter) if it is closed under the addition and stable under the product by arbitrary elements of $A$. An ideal $I \neq A$ is said to be prime if it satisfies the following condition:

$$
\text { If } a b \in I \text {, then } a \in I \text { or } b \in I \text {. }
$$

Finally, the spectrum of $A$ is the set $\operatorname{Spec} A$ of all prime ideals of $A$. 
Definition 2.2. The dimension of a distributive lattice $A$ is the supremum of all integers $n$ such that there exists a chain $P_{0} \subset P_{1} \subset \cdots \subset P_{n}$ of prime ideals in $A$. It may be infinite.

Definition 2.3. Let $X$ be a topological space. We say that a sublattice $B$ of $A_{X}$ is a closed basis, or just a basis, for the topology of $X$ if every closed set in $X$ is an intersection (possibly infinite) of closed subsets in $B$.

Definition 2.4. The arithmetical dimension of a topological space $X$ is the minimum of all integers $n$ such that there exists a basis for $X$ of dimension $n$. We denote it by $\operatorname{dim} X$.

Remarks. From now on, unless otherwise stated, the arithmetical dimension of a topological space $X$ will be referred to simply as the dimension of $X$.

It is very easy to prove that this dimension is monotone, i.e. if $Z$ is a subspace of $X$, then $\operatorname{dim} Z \leq \operatorname{dim} X$.

This dimension coincides with the graded dimension defined by J. Isbell [6], but the proof of the coincidence is not trivial.

Theorem 2.5 (Galián [4]). For separable metric spaces, the arithmetical dimension coincides with the inductive dimension and the covering dimension.

(2.6) For compact Hausdorff spaces, we have the following inequalities (see [6]) between the different dimension functions

$$
\text { cover } \operatorname{dim} X \leq \text { ind } X \leq \text { Ind } X \leq \operatorname{dim} X
$$

and there exists an example (Filippov [2]) of a compact Hausdorff space $X$ such that $1=\operatorname{cover} \operatorname{dim} X, 2=\operatorname{ind} X$ and $3=\operatorname{Ind} X$. Moreover (Filippov [3]), there exist compact Hausdorff spaces $X, Z$ such that $\operatorname{Ind} X=1, \operatorname{Ind} Z=2$ and $\operatorname{Ind}(X \times Z) \geq 4$. Since it is known (see [4, 10]) that $\operatorname{dim}(X \times Z) \leq$ $\operatorname{dim} X+\operatorname{dim} Z$, we conclude that Ind $K<\operatorname{dim} K$ for some compact Hausdorff space $K$.

Theorem 2.7 (Isbell [6]). $A T_{0}$ topological space has dimension $\leq n$ if and only if it is a subspace of an inverse limit of finite topological spaces of dimension $\leq n$.

Another proof of this theorem may be found in [9].

Example. We shall determine the dimension of the closed interval $[0,1]$. Let us consider the family $B$ of all finite unions of points and closed subintervals in $[0,1]$. It is clear that $B$ is a basis for the topology of $[0,1]$ and that any maximal ideal of $B$ has the form

$$
M_{x}=\{b \in B: x \in b\}, \quad \text { where } x \in[0,1] .
$$

Moreover, any nonmaximal prime ideal of $B$ has the form

$$
\begin{array}{ll}
P_{x}^{+}=\{b \in B:[x, x+\varepsilon] \subseteq b \text { for some } \varepsilon>0\}, & \text { where } 0 \leq x<1, \\
P_{x}^{-}=\{b \in B:[x-\varepsilon, x] \subseteq b \text { for some } \varepsilon>0\}, & \text { where } 0<x \leq 1,
\end{array}
$$


and we conclude that $\operatorname{dim} B=1$. Hence, the closed interval has dimension $\leq 1$. Now, the dimension of $[0,1]$ is 1 because zero-dimensional lattices are just Boolean algebras; therefore a space $X$ has dimension 0 if and only if its closed open subsets form a basis for $X$.

\section{Dense subalgebras of $C(X)$}

Definition 3.1. Let $A$ be an R-algebra. We say that a maximal ideal $M$ of $A$ is real if the canonical map $\mathbf{R} \rightarrow A / M$ is an isomorphism. The real spectrum of $A$ is the set $\operatorname{Spec}_{\mathrm{R}} A$ of all real maximal ideals of $A$.

Given a point $x \in \operatorname{Spec}_{\mathrm{R}} A$, the corresponding maximal ideal of $A$ will be denoted by $M_{x}$. Note that each element $f \in A$ defines a real function on the real spectrum by setting

$$
f(x)=\text { residue class of } f \text { in } A / M_{x}=\mathbf{R} .
$$

We shall always consider on $\operatorname{Spec}_{\mathbf{R}} A$ the initial (or weak) topology for these functions.

Definition 3.2. Let $X$ be a topological space and let $A$ be a subalgebra of the ring $C(X)$ of all real-valued continuous functions on $X$. We define a natural continuous map $j: X \rightarrow \operatorname{Spec}_{\mathbf{R}} A$ by setting

$$
j(x)=M_{x}=\{f \in A: f(x)=0\} .
$$

We shall say that $A$ separates points if for any two points $x, y \in X$ there exists $f \in A$ such that $f(x) \neq f(y)$. It is clear that $A$ separates points if and only if the natural map $j: X \rightarrow \operatorname{Spec}_{\mathbf{R}} A$ is injective.

Definition 3.3. Let $X$ be a topological space. We shall say that a subalgebra $A$ of $C(X)$ is basic if $X$ has the initial topology for the functions in the family A.

When $X$ is $T_{0}$, then $A$ is basic if and only if the natural map $j: X \rightarrow$ $\operatorname{Spec}_{\mathbf{R}} A$ is a homeomorphism of $X$ with the image $j(X)$.

We shall need the following reformulation of the Stone-Weierstrass theorem:

Theorem 3.4. Let $X$ be a compact Hausdorff space and let $A$ be a subalgebra of $C(X)$. Then the following conditions are equivalent:

(a) $A$ separates points.

(b) $A$ is basic.

(c) $A$ is dense in $C(X)$.

Proof. The equivalence (a) $\Leftrightarrow$ (c) is the usual statement of the Stone-Weierstrass theorem. The equivalence (a) $\Leftrightarrow(b)$ is very easy to prove.

Theorem 3.5. Let $A$ be a basic algebra of continuous functions on a compact Hausdorff space $X$ and let $A_{S}$ be the localization of $A$ with respect to the 
multiplicative system $S$ of all elements of $A$ which are invertible in $C(X)$. Then we have

$$
X=\operatorname{Spec}_{\mathrm{R}} A_{S} .
$$

Proof. It is clear that $A_{S}$ is a basic subalgebra of $C(X)$ and so it is enough to show that for each maximal ideal $M$ of $A_{S}$ there exists a point $x \in X$ such that $M=M_{x}=\left\{f \in A_{S}: f(x)=0\right\}$.

Let us now consider the closed subset $Z=\bigcap_{f \in M} f^{-1}(0)$. If $Z$ is nonempty, then we have $M \subseteq M_{x}$ for any $x \in Z$ and we obtain $M=M_{x}$ because $M$ is maximal. If $Z$ is empty, then we obtain a contradiction: since $X$ is compact, there must be elements $f_{1}, \ldots, f_{r} \in M$ such that $f_{1}^{-1}(0) \cap \cdots \cap f_{r}^{-1}(0)$ is empty, so that $f_{1}^{2}+\cdots+f_{r}^{2}$ belongs to $M$ and is invertible in $C(X)$ because its zero-set is empty. Hence, $M=A_{S}$ against the hypothesis of $M$ being a maximal ideal.

Remark 3.6. The proof actually shows that $X$ is the maximal spectrum of $A_{S}$ :

$$
X=\operatorname{Spec}_{\max } A_{S} .
$$

Moreover, if $A$ is an algebra of continuous functions on a compact Hausdorff space $X$ and $S$ is the multiplicative system of all elements of $A$ which are invertible in $C(X)$, then $\operatorname{Spec}_{\mathrm{R}} A_{S}=\mathrm{Spec}_{\max } A_{S}$ and this is a compact Hausdorff space such that we have a continuous projection

$$
j: X \rightarrow \operatorname{Spec}_{\mathbf{R}} A_{S}, \quad j(x)=M_{x}=\left\{f \in A_{S}: f(x)=0\right\} .
$$

\section{Characterization of the dimension}

Theorem 4.1 [10]. $\operatorname{dim}\left(\operatorname{Spec}_{\mathbf{R}} A\right) \leq \operatorname{dim} A$, for any $\mathbf{R}$-algebra $A$.

Proof. Let $B$ be the basis for the topology of $\operatorname{Spec}_{\mathrm{R}} A$ generated by the closed sets of the form $f^{-1}(I)$ where $f \in A$ and $I$ is a closed interval in the real line (infinite end points are admitted). We consider the natural map

$$
\operatorname{Spec} B \rightarrow \operatorname{Spec} A
$$

where the prime ideal $P^{\prime}$ of $A$ corresponding to a prime ideal $P$ of $B$ is just $P^{\prime}=\left\{f \in A: f^{-1}(0) \in P\right\}$. Now, to show that $\operatorname{dim} B \leq \operatorname{dim} A$, it is enough to prove that $P^{\prime}$ is strictly contained in $Q^{\prime}$ whenever $P$ is strictly contained in $Q$.

It is easy to check that $B$ is generated by the elements of the form $f^{-1}([0, \infty))$, so there exists $f \in A$ such that $f^{-1}([0, \infty)) \notin P$ and $f^{-1}([0, \infty)) \in Q$.

Since the zero of $B$ is the whole space $\operatorname{Spec}_{\mathrm{R}} A$, we have

$$
0=f^{-1}((-\infty, 0]) \cdot f^{-1}([0, \infty)) \quad(\text { recall } \cdot=\cup)
$$

and we get $f^{-1}((-\infty, 0]) \in P \subset Q$ because $P$ is prime. Therefore $f^{-1}(0)=$ $f^{-1}([0, \infty))+f^{-1}((-\infty, 0])$ belongs to $Q$ and $f^{-1}(0)$ does not belong to $P$ 
because otherwise we would obtain $f^{-1}([0, \infty))=f^{-1}([0, \infty)) \cdot f^{-1}(0) \in P$ against the choice of $f$. By definition, we conclude that $f \in Q^{\prime}$ and $f \notin P^{\prime}$, so that $P^{\prime}$ is strictly contained in $Q^{\prime}$.

Corollary 4.2. If $A$ is a basic algebra of continuous functions on a topological space $X$, then $\operatorname{dim} X \leq \operatorname{dim} A$.

Proof. Since $A$ is a basic subalgebra of $C(X)$, we have that $X$ is a subspace of $\operatorname{Spec}_{\mathrm{R}} A$; so $\operatorname{dim} X \leq \operatorname{dim}\left(\operatorname{Spec}_{\mathrm{R}} A\right)$ and we conclude by (4.1).

(4.3) Let $K$ be a finite triangulated polyhedron and let $V$ be the set of its vertices. Each map $V \rightarrow \mathbf{R}^{m}$ may be extended by linearity to a map $K \rightarrow \mathbf{R}^{m}$. We thus have a map $V \rightarrow \mathbf{R}^{n}, n=$ cardinality of $V$, that defines a natural immersion $K \rightarrow \mathbf{R}^{n}$.

Definition 4.4. A function on $K$ is said to be algebraic if it is the restriction of a polynomial function on $\mathbf{R}^{n}$. The algebra of all algebraic functions on $K$ will be denoted by $A(K)$.

Lemma 4.5. The ring $A(K)$ is a basic subalgebra of $C(K)$ such that $\operatorname{dim} K=$ $\operatorname{dim} A(K)$.

Proof. It is clear that $A(K)$ separates points and is therefore a basic subalgebra by (3.4).

On the other hand, we have $A(K)=\mathbf{R}\left[x_{1}, \ldots, x_{n}\right] / I$ where $I$ is the ideal of all polynomial functions vanishing on $K$. If $P_{s}$ denotes the prime ideal of all polynomial functions vanishing on a given closed simplex $s$ of $K$, then

$$
I=\bigcap_{s} P_{s}
$$

where $s$ runs over all the closed simplices of $K$; hence

$$
\operatorname{dim} A(K)=\operatorname{Max}_{s}\left\{\operatorname{dim} \mathbf{R}\left[x_{1}, \ldots, x_{n}\right] / P_{s}\right\} .
$$

Now, $P_{s}$ coincides with the ideal of all polynomial functions vanishing on the linear subspace spanned by $s$. A linear change of coordinates shows that we have $\mathbf{R}\left[x_{1}, \ldots, x_{n}\right] / P_{s}=\mathbf{R}\left[y_{1}, \ldots, y_{r}\right]$, where $r=\operatorname{dim} s$. We conclude that

$$
\operatorname{dim} A(K)=\operatorname{Max}_{s}\left\{\operatorname{dim} \mathbf{R}\left[x_{1}, \ldots, x_{n}\right] / P_{s}\right\}=\operatorname{Max}_{s}\{\operatorname{dim} s\}=\operatorname{dim} K .
$$

Definition 4.6. A continuous map $K \rightarrow L$ between two finite triangulated polyhedra is said to be semilinear if its restriction to each closed simplex of $K$ is linear.

If $K \rightarrow L$ is a semilinear map, then by composition it induces a morphism of R-algebras $A(L) \rightarrow A(K)$.

Lemma 4.7. Let $\left\{K_{i}, f_{i j}\right\}$ be an inverse system of finite triangulated polyhedra of dimension $\leq n$ and semilinear maps. Then there exists a basic algebra of continuous functions on $\operatorname{lm} K_{i}$ of dimension $\leq n$. 
Proof. Let us consider $A=\operatorname{lm}_{\vec{i}} A\left(K_{i}\right)$. One has that $\operatorname{dim} A \leq n$, because $\operatorname{dim} A\left(K_{i}\right) \leq n$ for any index $\vec{i}$ and the functor Spec takes direct limits into inverse limits.

Theorem 4.8. The dimension of any compact metric space $X$ is the minimum of the dimensions of all dense subalgebras of $C(X)$.

Proof. Let $n=\operatorname{dim} X$. By (4.2), we have $n \leq \operatorname{dim} A$ for any dense (= basic) subalgebra of $C(X)$. Hence, we must find a dense subalgebra of dimension $n$. By Freudenthal's Theorem, any compact metric space of dimension $\leq n$ is an inverse limit of finite triangulated polyhedra of dimension $\leq n$ and semilinear maps (see $[1,1.13 .2]$ ), so we conclude by (4.7).

Corollary 4.9. Let $X$ be a compact metric space of dimension $n$. Then there exists an n-dimensional dense subalgebra $A$ of $C(X)$ such that

$$
X=\operatorname{Spec}_{\mathbf{R}} A \text {. }
$$

Proof. By (4.8), there exists a dense subalgebra $A$ of dimension $n$. Localizing $A$ with respect to the multiplicative system of all its elements which are invertible in $C(X)$ we obtain the required dense subalgebra (see 3.5).

Corollary 4.10. A compact metric space has dimension $\leq n$ if and only if it is the real spectrum of an algebra of dimension $\leq n$.

Corollary 4.11. The dimension of any separable metric space $X$ is the minimum of the dimensions of all basic subalgebras of $C(X)$.

Proof. It is known $[1,1.7 .2]$ that any $n$-dimensional separable metric space $X$ has a compactification $X \rightarrow K$ where $K$ is a compact metric space of dimension $n$. By (4.8) there exists a basic subalgebra $A \subseteq C(K) \subseteq C(X)$ of dimension $n$ and, by (4.2), we know that $n \leq \operatorname{dim} A$ for any basic subalgebra A.

(4.12) The extension of Theorem 4.8 to arbitrary compact Hausdorff spaces would be a direct consequence of a positive answer to the following question:

Question. Let $X$ be a compact Hausdorff space of dimension $n$. Is $X$ a subspace of an inverse limit of $n$-dimensional finite triangulated polyhedra and semilinear maps?

Two facts suggest that the answer may be affirmative. First, Isbell's Theorem (2.7) stating that any $n$-dimensional $T_{0}$-space is a subspace of an inverse limit of $n$-dimensional finite spaces. Furthermore, each finite space gives rise to a finite triangulated polyhedron of the same dimension (named the geometric realization of the given finite space; see a canonical construction in [8 and 10]).

By (4.2) and (4.7), we have that any inverse limit of finite triangulated polyhedra of dimension $\leq n$ and semilinear maps has dimension $\leq n$; hence any subspace of such an inverse limit has dimension $\leq n$. Since there exists (see 2.6) a compact Hausdorff space $X$ such that $\operatorname{dim} X$ is greater than ind $X$, Ind $X$ 
and cover $\operatorname{dim} X$, we conclude that the answer to the question is negative when the arithmetical dimension is replaced by cover dim, ind or Ind.

Finally, we shall give a characterization of the covering dimension in terms of the Krull dimension of algebras of continuous functions (compare with Katětov's theorem, [5, 16.35]).

Theorem 4.13. The following conditions are equivalent for any compact Hausdorff space $X$ :

(a) The covering dimension of $X$ is $\leq n$.

(b) Every countably generated subalgebra of $C(X)$ is contained in the closure of a subalgebra of dimension $\leq n$.

Proof. (a) $\Rightarrow$ (b). Let $A$ be a countably generated subalgebra of $C(X)$ and let $S$ be the multiplicative system of all elements of $A$ which are invertible in $C(X)$. Then $K=\operatorname{Spec}_{\mathrm{R}} A_{S}=\operatorname{Spec}_{\max } A_{S}$ is a compact Hausdorff space and it has a countable basis because $A$ is countably generated, so it is a compact metric space. By Mardešić's Factorization Theorem [1, 3.3.2], the natural projection $j: X \rightarrow K$ factors through a compact metric space $\bar{K}$ of dimension $\leq n$

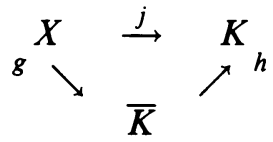

One thus has that $A \subseteq A_{S} \subseteq C(K) \stackrel{h^{*}}{\hookrightarrow} C(\bar{K}) \stackrel{g^{*}}{\hookrightarrow} C(X)$, where $h^{*}$ and $g^{*}$ are induced by composition with $h$ and $g$ respectively. By (4.8), we know that $C(\bar{K})$ is the closure of a subalgebra of dimension $\leq n$ and we may conclude.

(b) $\Rightarrow\left(\right.$ a). Let us consider all the countably generated subalgebras $B_{i} \subseteq$ $C(X)$, so that $C(X)=\operatorname{lm}_{\rightarrow} B_{i}$. By hypothesis, each subalgebra $B_{i}$ is contained in the closure of an algebra $A_{i}$ of dimension $\leq n$ and, localizing with respect to the multiplicative system of all elements which are invertible in $C(X)$, we may assume that $\operatorname{Spec}_{\mathrm{R}} A_{i}=\operatorname{Spec}_{\max } A_{i}$. Hence $K_{i}=\operatorname{Spec}_{\mathrm{R}} A_{i}$ is a compact metric space and $\operatorname{dim} K_{i} \leq n$ by (4.1). Let us now consider the natural projections $j_{i}: X \rightarrow K_{i}$. The closure of $A_{i}$ in $C(X)$ is therefore simply $C\left(K_{i}\right)$, via $j_{i}^{*}$. So we have

$$
C(X)=\lim _{\rightarrow} B_{i}=\lim _{\rightarrow} C\left(K_{i}\right)
$$

and it is clear that this fact implies that $X=\operatorname{lm} K_{i}$. We conclude, because we have

$$
\text { cover } \begin{aligned}
\operatorname{dim} X & \leq \operatorname{Max}_{i}\left\{\text { cover } \operatorname{dim} K_{i}\right\} \\
& =\operatorname{Max}_{i}\left\{\operatorname{dim} K_{i}\right\} \leq n .
\end{aligned}
$$

Remark 4.14. A standard argument extends Theorem 4.13 to arbitrary completely regular spaces, using $C^{*}(X)$ instead of $C(X)$. In this case the definition of the covering dimension must be modified so as to have cover $\operatorname{dim} X=$ cover $\operatorname{dim} \beta X$. 


\section{REFERENCES}

1. R. Engelking, Dimension theory, North-Holland Math. Library, vol. 19, North-Holland, Amsterdam, 1978.

2. V. V. Filippov, On bicompacta with noncoinciding inductive dimensions, Dokl. Akad. Nauk SSSR 192 (1970), 289-292; English transl., Soviet Math. Dokl. 11 (1970), 635-638.

3. $\ldots$, On the inductive dimension of the product of bicompacta, Dokl. Akad. Nauk SSSR 202 (1972), 1016-1019; English transl., Soviet Math. Dokl. 13 (1972), 250-254.

4. R. Galián, Teoria de la dimensión, Serie Univ. de la Fund. Juan March, no. 107, Madrid, 1979.

5. L. Gillman and M. Jerison, Rings of continuous functions, Graduate Texts in Math., no. 43, Springer-Verlag, Heidelberg, 1976.

6. J. Isbell, Graduation and dimension in locales, London Math. Soc. Lecture Notes Ser., no. 93, Cambridge Univ. Press, Cambridge, 1985, pp. 195-210.

7. M. Katětov, A theorem on the Lebesgue dimension, Časopis Pěst. Mat. Fys. 75 (1950), 79-87.

8. J. A. Navarro, Espacios topológicos finitos y homotopia, VIII. Jornadas Luso-Espanholas de Mat., Univ. de Coimbra, 1981, pp. 331-337.

9. J. B. Sancho and M. T. Sancho, Some results on dimension theory, Summer Meeting on Category Theory, Louvain-la-Neuve, 1987.

10. M. T. Sancho, Methods of commutative algebra for topology, Publ. Mat., no. 17, Univ. de Extremadura, Badajoz, 1987.

Departamento de Matemáticas, Universidad de Extremadura, Avenida de Elvas, 06071-BadaJoz, SPain

Departamento de Matemáticas, Universidad de Salamanca, Plaza de la Merced 1-4, 37008-Salamanca, Spain 